\title{
Application of optical coherence tomography for in vivo monitoring of the meningeal lymphatic vessels during opening of blood-brain barrier: mechanisms of brain clearing
}

Oxana Semyachkina-Glushkovskaya Arkady Abdurashitov Alexander Dubrovsky

Denis Bragin

Olga Bragina

Nataliya Shushunova

Galina Maslyakova

Nikita Navolokin

Alla Bucharskaya

Valery Tuchin

Juergen Kurths

Alexander Shirokov 


\title{
Application of optical coherence tomography for in vivo monitoring of the meningeal lymphatic vessels during opening of blood-brain barrier: mechanisms of brain clearing
}

\author{
Oxana Semyachkina-Glushkovskaya, ${ }^{a}$ Arkady Abdurashitov ${ }^{\mathrm{a}, \mathrm{b}, \mathrm{k}}$ Alexander Dubrovsky, ${ }^{\mathrm{a}, \mathrm{b}}$ Denis Bragin, ${ }^{\mathrm{a}, \mathrm{c}}$ \\ Olga Bragina, ${ }^{c}$ Nataliya Shushunova, ${ }^{a}$ Galina Maslyakova, ${ }^{d}$ Nikita Navolokin, ${ }^{\text {a,d }}$ Alla Bucharskaya, ${ }^{d}$ \\ Valery Tuchin, $, \mathrm{a}, \mathrm{b}, \mathrm{f}$ Juergen Kurths, ${ }^{\mathrm{a}, \mathrm{g}, \mathrm{h}}$ and Alexander Shirokov ${ }^{\mathrm{a}, \mathrm{i}, \mathrm{j}, \mathrm{k}}$ \\ ${ }^{a}$ Saratov State University, Interdisciplinary Center of Critical Technologies in Medicine, Saratov, Russia \\ 'Saratov State University, Department of Optics and Biophotonics, Saratov, Russia \\ 'University of New Mexico School of Medicine, Department of Neurosurgery, Albuquerque, New Mexico, United States \\ ${ }^{d}$ Saratov State Medical University, Department of Pathological Anatomy, Saratov, Russia \\ 'Tomsk State University, Laboratory of Biophotonics, Tomsk, Russia \\ fPrecision Mechanics and Control Institute of the Russian Academy of Sciences, Laboratory of Laser Diagnostics of Technical and Living Systems, \\ Saratov, Russia \\ gHumboldt University, Physics Department, Berlin, Germany \\ ${ }^{\text {h}}$ Potsdam Institute for Climate Impact Research, Potsdam, Germany \\ 'Institute of Biochemistry and Physiology of Plants and Microorganisms, Russian Academy of Sciences, Saratov, Russia \\ iSaratov State Medical University, Saratov, Russia \\ ${ }^{\mathrm{k}}$ Russian National Research Medical University, Moscow, Russia
}

\begin{abstract}
The meningeal lymphatic vessels were discovered 2 years ago as the drainage system involved in the mechanisms underlying the clearance of waste products from the brain. The blood-brain barrier (BBB) is a gatekeeper that strongly controls the movement of different molecules from the blood into the brain. We know the scenarios during the opening of the BBB, but there is extremely limited information on how the brain clears the substances that cross the BBB. Here, using the model of sound-induced opening of the BBB, we clearly show how the brain clears dextran after it crosses the BBB via the meningeal lymphatic vessels. We first demonstrate successful application of optical coherence tomography (OCT) for imaging of the lymphatic vessels in the meninges after opening of the BBB, which might be a new useful strategy for noninvasive analysis of lymphatic drainage in daily clinical practice. Also, we give information about the depth and size of the meningeal lymphatic vessels in mice. These new fundamental data with the applied focus on the OCT shed light on the mechanisms of brain clearance and the role of lymphatic drainage in these processes that could serve as an informative platform for a development of therapy and diagnostics of diseases associated with injuries of the BBB such as stroke, brain trauma, glioma, depression, or Alzheimer disease. () 2017 Society of Photo-Optical Instrumentation Engineers (SPIE) [DOI: 10.1117/1.JBO.22.12.121719]
\end{abstract}

Keywords: cerebral lymphatics; optical coherence tomography; blood-brain barrier.

Paper 170512SSR received Aug. 1, 2017; accepted for publication Dec. 4, 2017; published online Dec. 23, 2017.

\section{Introduction}

The meningeal lymphatic system that was recently discovered is a network of optically transparent vessels that play a vital role in the health of the central nervous system (CNS) in protecting the brain against pathogens. ${ }^{1-3}$ In 2015, Louveau et al. ${ }^{1}$ and Aspelund et $\mathrm{al}^{2}{ }^{2}$ clearly showed that the meningeal lymphatic system is involved in the clearing of waste products from the brain.

There is the hypothesis that the clearing function of the lymphatic system is one of the crucial mechanisms responsible for the CNS diseases related to injuries of the blood-brain barrier (BBB), which controls the transport of metabolic products to the brain. During the opening of the BBB, the metabolic and waste products, especially proteins, can pathologically accumulate in the brain and cause toxic effects on the brain tissues. For example, after stroke, which is strongly associated with the opening

*Address all correspondence to: Arkady Abdurashitov, E-mail: abdurashitovoptics@mail.ru of the BBB, the Evans Blue dye (EBd) complex with albumins $(68 \mathrm{kDa})$ penetrates through the $\mathrm{BBB}$, which is impossible in the normal state. ${ }^{4,5}$

The understanding the interaction between the lymphatic system and BBB functioning will open the door to progress into the effective prevention of vascular catastrophes in the brain associated with the disorders of the BBB. For instance, it can give a new impulse for early prognosis of stroke because the prestroke is accompanied by the opening of the BBB. ${ }^{6}$

However, there are some challenges to studying noninvasively the meningeal lymphatic system in vivo due to the optical transparency of lymphatic vessels (low scattering and absorption) and the low speed and nonstationary flow of lymph. There are some technical solutions including digital microscopy, ${ }^{7-9}$ speckle and Doppler imaging, ${ }^{7-9}$ photoacoustic, ${ }^{8,10-12}$ optical coherence tomography (OCT), ${ }^{13,14}$ molecular fluorescent, ${ }^{7,8,15}$ and near-infrared spectroscopy imaging. ${ }^{16}$ Note that all these 
methods were used only for the analysis of the peripheral lymphatic vessels, but they are significantly larger and have a wider network than the meningeal lymphatic vasculature, which makes them more easily visible. The development of new strategies for noninvasive study of the meningeal lymphatic vessels can give impulse for the application of diagnostic methods in practical medicine, in particular, for the prognosis of subdural intracranial hemorrhages in newborns that are associated with the injuries of the brain drainage system. ${ }^{17}$

Here, for the first time, we show the successful application of OCT in noninvasive imaging of the meningeal lymphatic system in our study of lymphatic drainage after opening of the BBB.

\section{Methods}

\subsection{Subjects}

Male mice (20 to $25 \mathrm{~g}, n=89$ ) were used in all experiments. The animals were housed under standard laboratory conditions, with access to food and water, ad libitum. All procedures were performed in accordance with the "Guide for the Care and Use of Laboratory Animals." The experimental protocol was approved by the Committee for the Care and Use of the Laboratory Animals at the Saratov State University (Protocol H-147, 17.04.2001).

\subsection{Assessment of the BBB Permeability}

Four different techniques were used to evaluate the BBB disruption:

i. A spectrofluorometric assay of EBd extravasation: Polyethylene catheter (PE-10 tip, Scientific Commodities Inc., Lake Havasu City, Arizona) was inserted into the right femoral vein for EBd (Sigma Chemical Co., St. Louis, Michigan) intravenous injection in a single bolus dose $(2 \mathrm{mg} / 25 \mathrm{~g}$ mouse, $1 \%$ solution in physiological $0.9 \%$ saline). The implantation of the catheter was performed under inhalation anesthesia (2\% isoflurane, $70 \% \mathrm{~N}_{2} \mathrm{O}$, and $30 \% \mathrm{O}_{2}$ ). The EBd circulated in the blood for $30 \mathrm{~min}$ in accordance with the recommended protocol. ${ }^{18}$ Then, the mice were decapitated, and their brains were quickly collected and placed on ice (no anticoagulation was used during blood collection). The detailed protocol of EBd extraction and visualization was published by Wang et al. ${ }^{19}$ Here is a brief description of this method. Prior to brain removal, the brain was perfused with $0.9 \%$ saline to rid the circulating remaining dye in the cerebral vessels. The isolated brains were cut into small pieces and incubated with $0.9 \%$ saline (1:3) for $60 \mathrm{~min}$ to enable the soluble substances to dissolve. Then the solutions were centrifuged at $10,000 \mathrm{~g}$ for 10 min to sediment the nondissolved parts. The supernatants that contained the brain solutes were treated by $50 \%$ trichloroacetic acid (TCA; 1:3) and centrifuged again at $10,000 \mathrm{~g}$ for $20 \mathrm{~min}$ to remove precipitated molecules. The final supernatant was incubated in $95 \%$ ethanol to increase the optic signals for spectrofluorimetry assay (620 nm/680 nm, Agilent Cary Eclipse, Agilent). The standard calibration curve was used to measure the EBd concentration ( $\mu \mathrm{g} / \mathrm{g}$ of brain tissues).

The analysis of EBd extravasation was performed in the groups (1) before sound (the control, $n=7$ ) and after sound: (2) $1 \mathrm{~h}(n=7), 4 \mathrm{~h}(n=7)$, and $24 \mathrm{~h}(n=7)$. ii. A confocal microscopy of FITC-dextran $70 \mathrm{kDa}$ extravasation: The protocol for high weight fluorescein isothiocyanate (FITC)-dextrans extravasation to assess BBB disruption using a confocal microscopy is described in detail. ${ }^{18}$ Here is a brief description of the main steps of this protocol. FITC-dextran $70 \mathrm{kDa}$ was injected intravenously ( $4 \mathrm{mg} / 25 \mathrm{~g}$ mouse, $0.5 \%$ solution in $0.9 \%$ physiological saline, Sigma) and circulated for $20 \mathrm{~min}$. Afterward, the mice were decapitated, and their brains were quickly removed and fixed in $4 \%$ paraformaldehyde (PFA) for $24 \mathrm{~h}$, cut into $50-\mu \mathrm{m}$ thick slices on vibratome (Leica VT 1000S Microsystem, Germany) and analyzed on a confocal microscope (Olympus FV10i-W, Olympus, Japan). Approximately 8 to 12 slices per animal from the cortical and subcortical (excepting the hypothalamus and choroid plexus, where the $\mathrm{BBB}$ is leaky) regions were imaged. The confocal microscopy of the BBB permeability was performed in the groups (1) before sound (the control, $n=7)$ and after sound: (2) $1 \mathrm{~h}(n=7), 4 \mathrm{~h}(n=7)$, and $24 \mathrm{~h}(n=7)$.

iii. A histological analysis of BBB permeability to solutes: This experiment was performed in the same groups that we used for confocal imaging with dextran. All mice were decapitated after the performed experiments. The samples were fixed in $10 \%$ buffered neutral formalin. The formalin fixed specimens were embedded in paraffin, sectioned ( $4 \mu \mathrm{m})$, and stained with haematoxylin and eosin. The histological sections were evaluated by light microscopy using the digital image analysis system Mikrovizor medical $\mu$ Vizo-103 (LOMO, Russia).

iv. In vivo real-time two-photon laser scanning microscopy (2PLSM): The BBB permeability was continuously monitored by measuring the perivascular tissue fluorescence in seven mice at different time points: before and 1 to 4 to $24 \mathrm{~h}$ after sound exposure as described previously with some modifications. ${ }^{20}$ Three days before imaging, chronic optical windows $(\phi 3 \mathrm{~mm})$ were prepared in coordinates 1 to $4 \mathrm{~mm}$ caudal and 1 to $4 \mathrm{~mm}$ lateral to bregma as we previously described. ${ }^{20}$

During the imaging, mice were kept under inhalation anesthesia with $2 \%$ isoflurane at $1 \mathrm{~L} / \min \mathrm{N}_{2} \mathrm{O} / \mathrm{O}_{2}-70: 30$. The body temperature was maintained at $37.5^{\circ} \mathrm{C}$ by a homoeothermic blanket system with rectal probe (Harvard Apparatus, Holliston, Massachusetts). The brain temperature was kept at $37^{\circ} \mathrm{C}$ using an objective heating system with a temperature probe (Bioptechs Inc., Butler, Pennsylvania). The fluoresceinlabeled dextran (70 kDa, Sigma-Aldrich) in physiological saline (5\% wt/vol) was injected through the tail vein $(\sim 100 \mu \mathrm{l})$ at an estimated initial concentration in blood serum of $150 \mathrm{Mm} .^{21}$

Imaging was done using Prairie View Ultima system with an Olympus BX51WI upright microscope and water-immersion LUMPlan FL/IR $20 \times / 0.50 \mathrm{~W}$ objective. The excitation was provided by a Prairie View Ultima multiphoton laser scan unit powered by a Millennia Prime $10 \mathrm{~W}$ diode laser source pumping a Tsunami Ti:sapphire laser (Spectra-Physics) tuned up to $810 \mathrm{~nm}$ center wavelength. Fluorescence was bandpass filtered at 510 to $550 \mathrm{~nm}$. Real time images $(512 \times 512$ pixels, $0.15 \mu \mathrm{m} /$ pixel in the $x$ - and $y$-axes) were acquired using the time series mode of the Prairie View software (30-s intervals between images, $30 \mathrm{~min}$ sessions). Between the sessions (before and 
1 to 4 to $24 \mathrm{~h}$ after sound exposure) mice were awake and were kept at the animal facility.

In offline analyses using ImageJ, microvascular BBB permeability was evaluated by measuring changes in perivascular tissue fluorescence in planar images of the cortex taken 50 and $150 \mu \mathrm{m}$ depth $20 \mathrm{~min}$ after fluorescein dextran injection, as described previously. ${ }^{20}$ On each image, fluorescence of 10 randomly chosen regions of interest over the vessel areas and 10 corresponding perivascular brain parenchyma areas were measured. The obtained measurements in the interstitial space were normalized to the maximal fluorescence intensity in the blood vessels and expressed as a percentage of fluorescence intensity (modified technique from Egawa et al. ${ }^{22}$ ). The estimated quantity of fluorescein that leaked out of the vessel and left in the vessel $20 \mathrm{~min}$ after the injection was calculated from the initial vascular fluorescence equal to $\sim 150 \mu \mathrm{M}$ of fluorescein. The time courses of fluorescein leakage for each group were also plotted.

To image the meningeal lymphatic vessels, 2PLSM was performed as described above with some modifications. Just before 2PLSM imaging, mice were preanesthetized in a box with $5 \%$ isoflurane in $\mathrm{N}_{2} \mathrm{O} / \mathrm{O}_{2}-50: 50$, secured on stereotactic frame, and switched to inhalation anesthesia with $2 \%$ isoflurane at $1 \mathrm{~L} / \mathrm{min} \mathrm{N}_{2} \mathrm{O} / \mathrm{O}_{2}-70: 30$. To label the lymphatic vessels, mice were injected i.c.v. (into the cisterna magna) with $5 \mathrm{ml}$ of Alexa 488-conjugated anti-Lyve-1 antibody (ALY7, eBioscience) as described in Ref. 1 and moved to the 2PLSM setup. After baseline imaging, mice underwent sound treatment, and imaging was repeated at $1 \mathrm{~h}$ after the end of sound treatment. In offline analysis, using a National Institutes of Health, diameters of the lymphatic vessels were quantified.

\subsection{Optical Coherence Tomography}

The OCT imaging of meningeal lymphatic vessels was performed with the commercial OCT system (Thorlabs Ganymede) providing lateral and transverse resolution of this system is $6.2 \mu \mathrm{m}$ (on air) and $8 \mu \mathrm{m}$, respectively, with the corresponding 1.3 and $3 \mu \mathrm{m}$ pixel size in the resulting image. The central wavelength of the OCT light source is $930 \mathrm{~nm}$. Each B-scan consists of 768 A-lines. Contrast enchantment was done by equalizing the histograms of the images. Due to the fact that the lymph is optically transparent within a broad range of wavelengths, in the resulting B-mode OCT image, the lymphatic vessels will look like an "empty" tube with the inverted signal-to-noise ratio regarding background; this is a so-called "negative" contrast. The main feature of the meningeal lymphatic vessels is the lack of shadowing and decorrelation "tail" beneath them during the lack of high scattering turbid media (please see Video 1 for details). These two features allow us to easily identify optically resolvable vessels inside the tissue.

The OCT imaging was performed in the groups (1) before sound (the control, $n=5)$ and $1 \mathrm{~h}$ after sound $(n=7)$.

\subsection{Immunohistochemical Analysis}

For the visualization of the meningeal lymphatic vessels, we used the protocol for the immunohistochemical (ICH) analysis with specific marker LYVE1 - the lymphatic vessel endothelial hyaluronan receptor $1 .{ }^{23-25}$ Tissue samples were fixed with formaldehyde and, after routine processing, were embedded into a paraffin block. Then samples were sectioned into 3- to $5-\mu \mathrm{m}$ sections; the sections were attached to poly-L-lysinecoated glass slides; they were dried at $37^{\circ} \mathrm{C}$ for $24 \mathrm{~h}$; and they were sequentially incubated in xylene (three times, $3 \mathrm{~min}$ each), 96\% ethanol (three times, 3 min each), 80\% ethanol (two times, $3 \mathrm{~min}$ each), and distilled water (three times, $3 \mathrm{~min}$ each). The IHC reaction was visualized with a REVEAL-BiotinFree Polyvalent diaminobenzidine kit (Spring Bioscience). Endogenous peroxidases were blocked by adding $0.3 \%$ hydrogen peroxide to the sections for $10 \mathrm{~min}$ followed by washing the sections in phosphate buffered saline (PBS). The antigen retrieval was conducted using a microwave oven in an ethylenediaminetetraacetic acid-buffer $\mathrm{pH}$ 9.0, and nonspecific background staining was blocked in PBS containing $0.5 \%$ bovine serum albumin and $0.5 \%$ casein for $10 \mathrm{~min}$, after which the sections were washed in PBS for $5 \mathrm{~min}$. Further, the sections were incubated in a humid chamber with diluted anti-LYVE1 antibody $(1: 1000)$ for $1 \mathrm{~h}$ at room temperature. After that, the sections were washed in PBS, incubated with secondary horseradish peroxidase-labeled goat antirabbit antibodies for 15 min, again washed in PBS, counterstained with hematoxylin for $1 \mathrm{~min}$, washed in water, dehydrated in graded alcohols (three times, 3 min each) and then in xylene (three times, 3 min each), and finally embedded into Canadian balm.

The ICH analysis was performed after the OCT imaging in the same groups.

\subsection{Confocal Imaging of Lymphatic Drainage after the Opening of BBB}

To induce opening of the BBB, we used an audible sound $(110 \mathrm{~dB}, 370 \mathrm{~Hz}): 60 \mathrm{~s}$ sound, then $60 \mathrm{~s}$ pause, and this cycle was repeated for $2 \mathrm{~h}^{26}$ This procedure was performed using a sound transducer in a Plexiglas ${ }^{\circledR}$ chamber (with volume of $2000 \mathrm{~cm}^{3}$ ) amplifying the effect of sound on the mice and not passing sound out.

To study the lymphatic drainage, we used the following experimental design: the mice underwent by sound during $2 \mathrm{~h}$; then, $1 \mathrm{~h}$ after sound when the BBB opened, FITC-dextran $70 \mathrm{kDa}$ was injected intravenously $(1 \mathrm{mg} / 25 \mathrm{~g}$ mouse, $0.5 \%$ solution in $0.9 \%$ physiological saline, Sigma-Aldrich) and allowed to circulate for $20 \mathrm{~min}$. Afterward mice were anaesthetized by ketamine/xylazine injection i.p. and injected i.c.v. (into the cisterna magna) with $5 \mathrm{ml}$ of Alexa488-conjugated anti-Lyve-1 antibody (ALY7, eBioscience). Ten minutes after this procedure, the mice were decapitated; their brains were quickly removed, fixed in $4 \%$ PFA for $24 \mathrm{~h}$, cut into $50-\mu \mathrm{m}$ thick slices on vibratome (Leica VT 1000S Microsystem, Germany), and analyzed on a confocal microscope (Olympus FV10i-W, Olympus, Japan). Approximately 8 to 12 slices per animal were imaged. The confocal imaging was performed in the groups (1) before sound (control, $n=7$ ) and $1 \mathrm{~h}$ after sound $(n=7)$.

\subsection{Statistical Analysis}

The results are presented as a mean \pm standard error of the mean (SEM). Differences from the initial level in the same group were evaluated by the Wilcoxon test. Intergroup differences were evaluated using the Mann-Whitney test and twoway analysis of variance (post hoc analysis with the Duncan's rank test). Significance levels were set at $p<0.05$ for all analyses. 


\section{Results}

\subsection{Sound-Induced Opening of BBB}

In the first step of our study, we analyzed the BBB permeability to EBd and FITC-dextran as a classical test for BBB integrity.

Confocal microscopy revealed the accumulation of FITCdextran outside of the brain capillaries at $1 \mathrm{~h}$ after sound exposure. Extravasation of FITC-dextran was determined as a clear fluorescence visualized outside of the vessel walls. Vascular leakage of FITC-dextran was defined as bright red clouds associated with a group of capillaries [Fig. 1(b)]. At 4 and $24 \mathrm{~h}$ after sound, the BBB rapidly recovered, which was accompanied by closing of the BBB to FITC-dextran. No extravasation of FITCdextran was observed in the control group (no sound) [Fig. 1(a)].

2PLSM of FITC-dextran leakage, injected intravenously, confirmed the results obtained ex vivo using confocal microscopy imaging. The analysis of microvascular permeability to FITC-dextran using 2PLSM was performed on each of 7 mice before (no) and 1, 4, and $24 \mathrm{~h}$ after the sound exposure. Indeed, 2PLSM imaging showed an impaired BBB $1 \mathrm{~h}$ after the sound and the normalization of BBB permeability to FITCdextran 4 and $24 \mathrm{~h}$ after the sound exposure [Fig. 1(c)].
Leakage of EBd also significantly increased $1 \mathrm{~h}$ after sound exposure [Fig. 1(d)]. Indeed, $1 \mathrm{~h}$ after the sound, a 19.7-fold increase in the leakage of EBd was observed $(7.3 \pm 0.09$ versus $0.37 \pm 0.02, \mu \mathrm{g} / \mathrm{g}$ of tissue, $p<0.05$ ). Four hours and twentyfour hours after the sound, the BBB permeability to EBd was completely normalized and the level of dye in the brain tissues was similar to the normal state $(0.54 \pm 0.01, \mu \mathrm{g} / \mathrm{g}$ of tissue and $0.40 \pm 0.03, \mu \mathrm{g} / \mathrm{g}$ of tissue, respectively).

Thus, in the first series of experiments, we clearly show that sound causes a temporal opening of the BBB with maximal effects $1 \mathrm{~h}$ after sound exposure.

\subsection{Visualization of the Meningeal Lymphatic Vessels before and after the Opening of BBB}

In the second step, we analyzed the interaction between the meningeal lymphatic vessels and the increasing of BBB permeability. Following this task, we analyzed the changes in diameter of the meningeal lymphatic vessels in three independent groups using in vitro IHC analysis and in vivo OCT and 2PLSM.

OCT and 2PLSM data show similar depths of the meningeal lymphatic vessels, which is 30 to $50 \mu \mathrm{m}$ from the surface of the
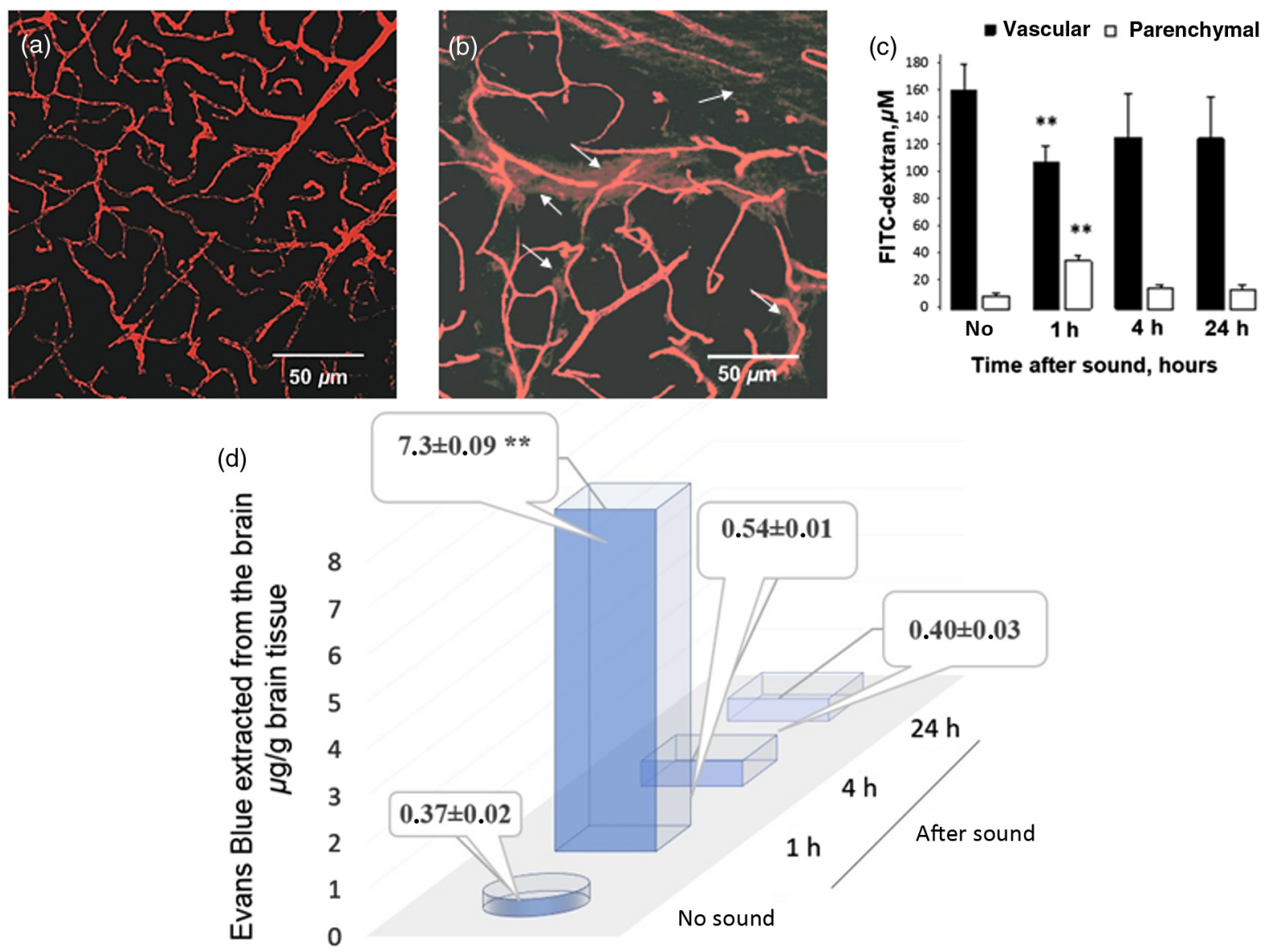

Time after sound, hours

Fig. 1 BBB permeability to FITC-dextran $70 \mathrm{kDa}$ and EBd with albumin complex $68 \mathrm{kDa}$ : (a) before sound exposure (no extravasation of FITC-dextran); (b) $1 \mathrm{~h}$ after sound exposure [strong extravasation of FITC-dextran (arrowed), which was defined as bright red clouds associated with a group of capillaries]; (c) the estimation of FITC-dextran concentration in brain parenchyma and microvessels 20 min after the injection. 2PLSM data are presented as mean \pm SEM, $n=7,{ }^{* *} p<0.01$; (d) EBd in $\mu \mathrm{g} / \mathrm{g}$ of mouse brain tissue before and after sound. Spectrofluorometric assay of EBd extravasation into the brain parenchyma shows an increase in the BBB permeability to EBd $1 \mathrm{~h}$ after the sound with rapid restoring of the BBB afterward. 
(a)

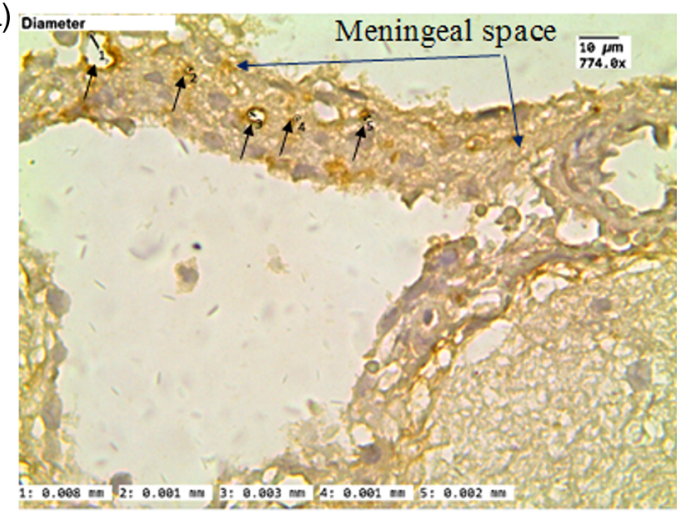

(c)

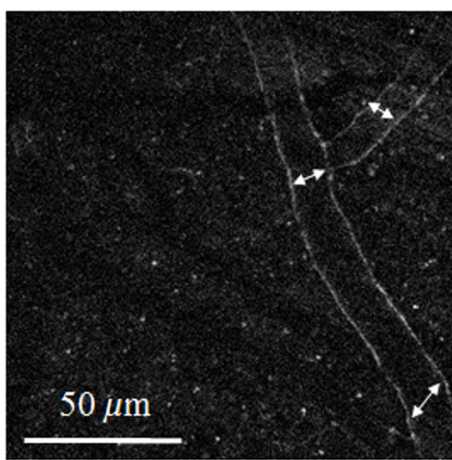

(b)

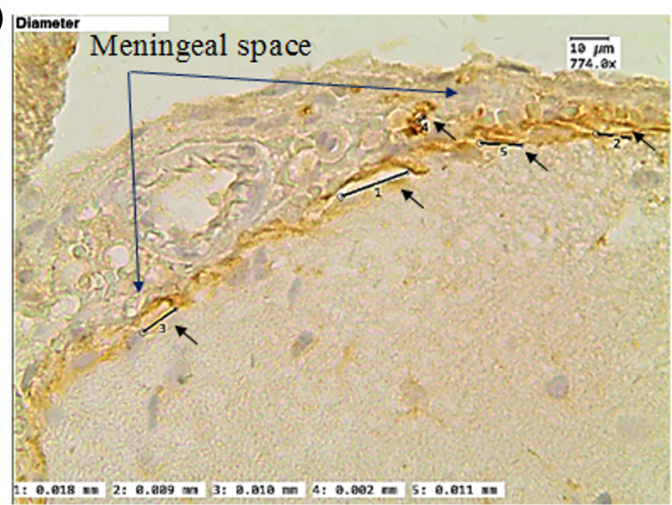

(d)

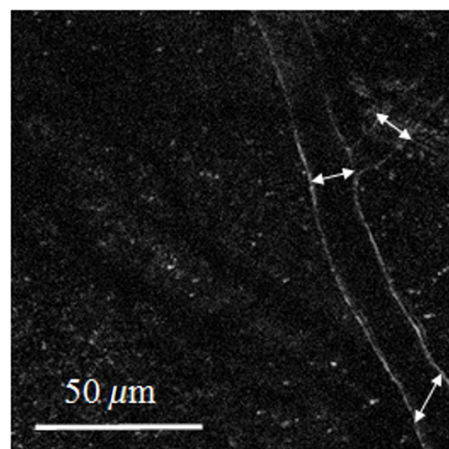

Fig. $2 \mathrm{ICH}(\mathrm{a}$ and $\mathrm{b}$ ) and in vivo two-photon laser scanning (c and d) visualization of the meningeal lymphatic vessels: (a) and (b) the meningeal lymphatic vessels stained with LYVE1 (specific marker of endothelial cells of lymphatic vessels, the brown color is positive staining); (a) before sound-induced opening of the BBB, the average diameter of lymphatic vessels is $3.00 \pm 0.01 \mu \mathrm{m}$; (b) after opening of the BBB (1 $\mathrm{h}$ after sound), the average diameter of some lymphatic vessels is $10.00 \pm 0.07 \mu \mathrm{m}$; (c) and (d) the meningeal lymphatic vessels before and after opening of the BBB, respectively. 2PLSM data show the increase of diameters of the lymphatic vessels after opening of the BBB: $(12.00 \pm 0.03 \mu \mathrm{m}$ versus $17.00 \pm 0.08 \mu \mathrm{m}, p<0.05)$.

brain, i.e., superficially in the meningeal layers of the brain [Figs. 2(b) and 3(c)].

The IHC and 2PLSM analysis revealed that in the control group (before opening of the BBB) the average diameter of the meningeal lymphatic vessels is $3.00 \pm 0.01 \mu \mathrm{m}$ (IHC data) and $12.00 \pm 0.03 \mu \mathrm{m}$ (2PLSM data). When the BBB permeability increased (1 $\mathrm{h}$ after sound), some of the lymphatic vessels became larger with the average diameter-10.00 $\pm 0.07 \mu \mathrm{m}$ (IHC data) and $17.00 \pm 0.08 \mu \mathrm{m}$ (2PLSM data) [Figs. 2(a)-2(d)]. The differences in the size of the diameter of the meningeal lymphatic vessels can be explained by different regions of the brain taken for the analysis. Technically 2PLSM was performed in the area of superior sagittal sinus, an unpaired large venous channel between the endosteal and meningeal layers of dura mater that runs in a sagittal plane from the anterior aspect of the falx cerebri to its termination at the confluence of sinuses at the occipital protuberance. The IHC data were obtained from the area of sigmoid sinus, which begins beneath the temporal bone and follows a tortuous course to the jugular foramen, at which point the sinus becomes continuous with the internal jugular vein. The different sizes of the meningeal lymphatic vessels were also discussed by Aspelund et al. ${ }^{2}$ Another possible reason is brain fixation, performed before brain harvesting, which could cause the lymphatic vessels to shrink.

The OCT can image the meningeal lymphatic vessels only after opening of the BBB but not in the normal state due to the very small size of the lymphatic vessels in the norm. Figures 3(a) and 3(c) show the OCT images of the meningeal lymphatic vessel (black empty spaces) surrounding the sagittal and sigmoid sinuses; the average diameter of the visible lymph vessels after the increase of $\mathrm{BBB}$ permeability is $-19.00 \pm 0.11 \mu \mathrm{m}$. Video 1 shows the decorrelation speckle beneath the blood vessels, which are close to the meningeal lymphatic vessels.

To be sure that we visualize the meningeal lymphatic vessels by the OCT, which look as a black empty space, we analyzed the size of the perivascular space (PVS), which also can potentially give a dark space on the OCT, especially after opening of the $\mathrm{BBB}$ when fluids move from the vessels into the PVS. Figure 4 shows that the PVS do not change significantly $1 \mathrm{~h}$ after the sound-induced opening of the BBB compared with the control. Due to this reason, we cannot detect the PVS in mice using 2PLSM in both the normal state and after opening of the BBB. The size of PVS is smaller than the black empty space on the OCT $(3.00 \pm 0.03 \mu \mathrm{m}$ versus $19.00 \pm 0.11 \mu \mathrm{m}$, respectively), which allows us to conclude that the black empty space is not the PVS or the PVS can be included only partly in the OCT imaging. Additionally, on the Video 1, we clearly show the differences between the OCT signals from the cerebral vein and the meningeal lymphatic vessels.

\subsection{Lymphatic Drainage after the Opening of BBB}

Since in our previous steps of study we observed increasing of diameter of the meningeal lymphatic vessels after opening of the 
(a)

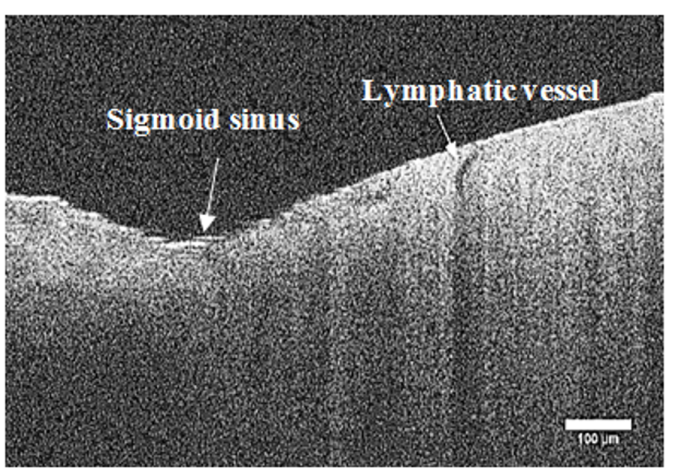

(c) 120.00

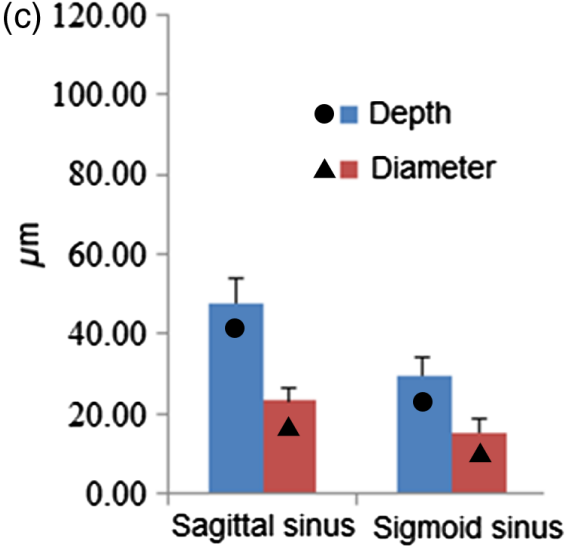

(b)

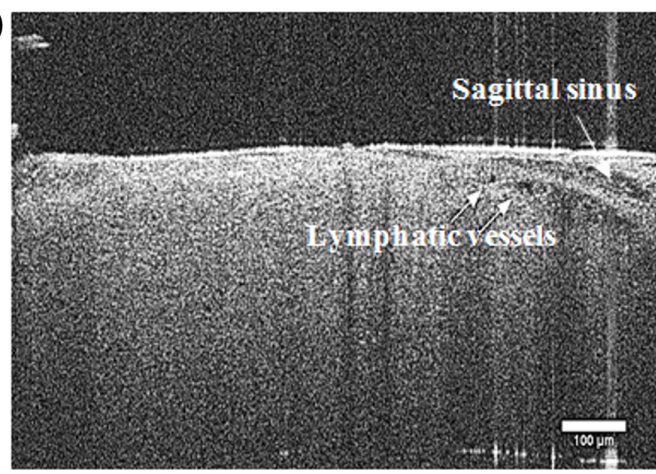

(d)

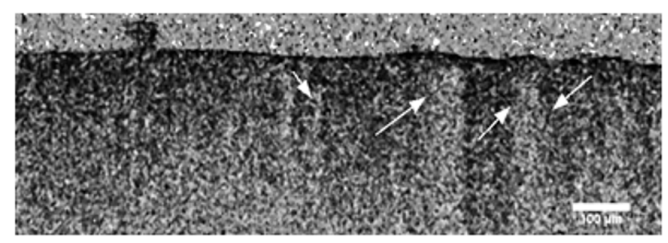

Fig. 3 The OCT images of the meningeal lymphatic vessels (black empty spaces) surrounding the sigmoid (a) and sagittal (b) sinuses. (c) The OCT analysis of diameter and depth of the meningeal lymphatic vessels. Scale bar is $100 \mu \mathrm{m}$ (Video 1, MP4, 7.07 MB [URL: http://dx.doi.org/10.1117/1.JBO.22.12 $.121719 .1])$.

(a)

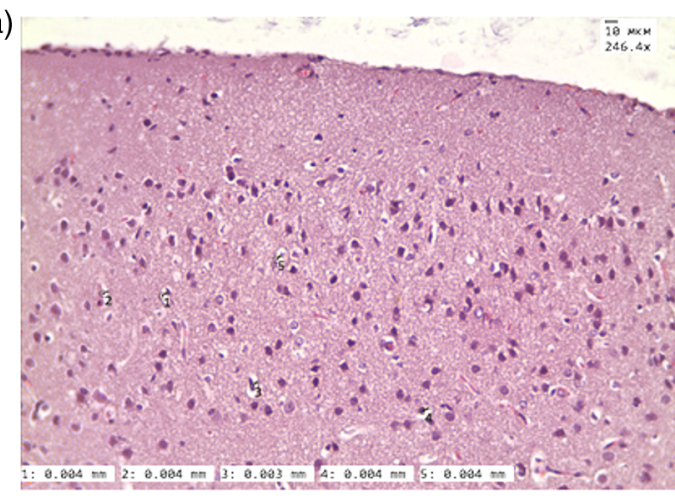

(b)

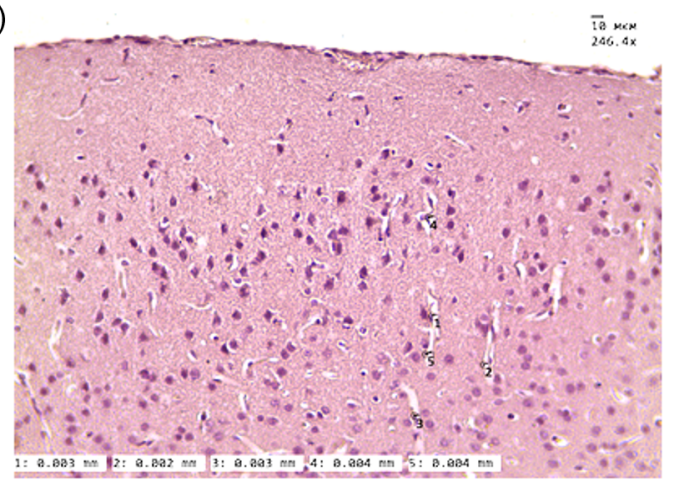

Fig. 4 The histological analysis of size of PVS (a) before and (b) $1 \mathrm{~h}$ after sound-induced opening of the BBB $3.8 \mu \mathrm{m}-$ in the normal state and $3.2 \mu \mathrm{m} 1 \mathrm{~h}$ after the opening of the BBB.

BBB, we hypothesized that it can be associated with the drainage and clearing function of lymphatics. To test our hypothesis, we tried to answer the question, "If we open the BBB for dextran, is it possible to see this fluorescent tracer in the meningeal lymphatic vessels as one of the possible ways for its clearing from the brain?"

To answer this question, mice underwent sound during $2 \mathrm{~h}$. Then $1 \mathrm{~h}$ after sound when the BBB opened, FITC-dextran was injected intravenously and circulated for $20 \mathrm{~min}$. Then, the mice were anaesthetized and injected into the cisterna magna with Alexa488-conjugated anti-Lyve-1 antibody for the labeling of the meningeal lymphatic vessels. Ten minutes after this procedure, the mice were decapitated, and their brains were quickly removed for the confocal microscopy protocol. Thus, $30 \mathrm{~min}$ was the time for FITC-dextran extravasation through BBB, its accumulation in PVS and parenchyma, and then for clearance.

Figure 5 shows representative confocal image of the meningeal lymphatic vessels after opening of the BBB. Our results clearly demonstrate the presence of FITC-dextran directly in the meningeal lymphatic vessels after opening of the BBB.

\section{Discussion}

The meningeal lymphatic system is an intriguing field in neuroscience. The lymphatic vessels in the meninges were clearly visualized only 2 years ago. ${ }^{1,2}$ Therefore, the information about 


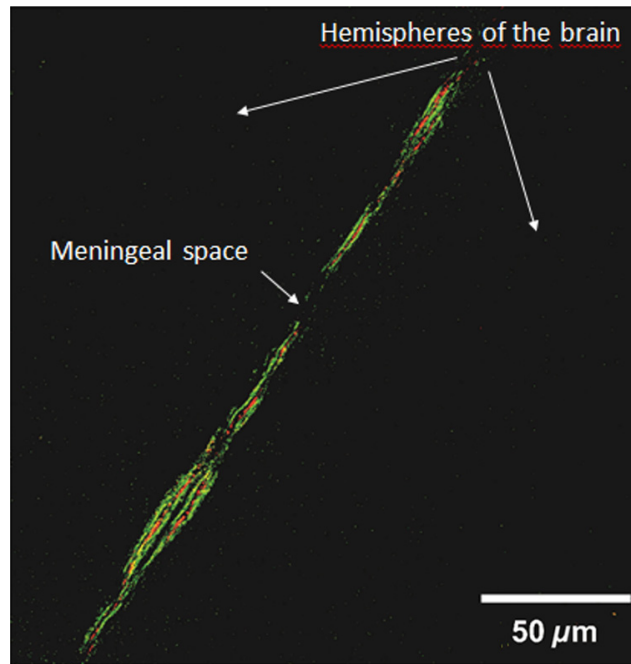

Fig. 5 The confocal image of meningeal lymphatic vessels after the sound-induced opening of the BBB: the dextran (red color) is obseved in the meningeal lymphatic vessels (green color) 30 min after the opening of the BBB.

the role of this system in brain functioning is extremely limited. 2PLSM and confocal imaging are optical techniques that are successfully used for the experimental studies of the meningeal lymphatic system. For the effective application of new fundamental knowledge about the meningeal lymphatics, it is necessary to develop further tools that are suitable for clinical use.

One such optical tool is the OCT, which we successfully used here for visualization of the meningeal lymphatic vessels in the study of the lymphatic drainage after sound-induced opening of the BBB. The ICH and 2PLSM analysis revealed a small size of the meningeal lymphatic vessels in the normal state $(3.00 \pm 0.01 \mu \mathrm{m}$ in the area of sigmoid sinus, IHC data, and $12.00 \pm 0.03 \mu \mathrm{m}$ in the area of sagittal sinus, 2PLSM data). This is the reason why we did not see these vessels in intact mice using OCT as it has lower spatial resolution. Note that OCT has been applied for the imaging of the cutaneous lymphatic vessels in human burn scars when the lymphatic vessels become enlarged due to accumulation of fluid in injured tissues. ${ }^{14}$

To be sure that we visualize exactly the meningeal lymphatic vessels by OCT, which look as black empty space, we analyzed the size of the PVS. The PVS can potentially give a dark space on OCT after opening of the BBB associated with the fluid movement from the cerebral vessels into the parenchyma. Our histological data clearly show that the size of PVS is smaller than the black empty space on OCT, which allows us to conclude that the black empty space is not the PVS or the PVS can be included only partly in OCT imaging.

Our OCT data presented on the depth of 30 to $50 \mu \mathrm{m}$, i.e., in the meninges, while the PVS surrounds the wall of arteries and arterioles (Virchow-Robin space), veins, and venules (perivenular space) from the subarachnoid space along their intraparenchymatous course.

According to the hypothesis of Alexander Monro and George Kellie, the cranium and its constituents (blood, cerebrospinal fluid, and brain tissue) create a state of volume equilibrium such that any increase in volume of one of the cranial constituents must be compensated by a decrease in volume of another. ${ }^{27} \mathrm{We}$ suppose that after opening of the BBB the compensatory mechanisms of distribution of cerebral blood flow are involved; therefore, we cannot see the enlarged PVS. ${ }^{26}$
The opening of the BBB is associated with the increase of diameter of the meningeal lymphatic vessels (the IHC and 2PLSM data). Indeed, the IHC data suggest that the diameter of these vessels is a 3.3-fold larger $1 \mathrm{~h}$ after opening of the BBB compared with one in the normal state $(3.00 \pm 0.01 \mu \mathrm{m}$ versus $10.00 \pm 0.07 \mu \mathrm{m}, p<0.05)$, according to the 2 PLSM data 1.5 -fold $(12.00 \pm 0.03 \mu \mathrm{m}$ versus $17.00 \pm 0.03 \mu \mathrm{m}$, $p<0.05)$. We also observed the presence of dextran in the meningeal lymphatic vessels $30 \mathrm{~min}$ after its extravasation through the BBB. Our results are consistent with data of other researchers, who also showed the clearance of different dyes from the brain via a lymphatic pathway. ${ }^{28-30}$ However, in these classical works, the dyes were injected directly into the brain parenchyma and their clearance observed in the deep cervical lymph nodes apart to the site of injection. They explained these results as the movement of the cerebral spinal fluid from the subarachnoid space into the nasal lymphatics through the cribriform plate due to the absence of knowledge about lymphatics in the brain. ${ }^{28-31}$ Pioneer studies of Louveau et al. ${ }^{1}$ and Aspelund et al. $^{2}$ changed this dogma and showed the direct role of the meningeal lymphatic system in the clearance of dyes from the brain. ${ }^{1,2}$

Here, using an original design of experiments, for the first time, we showed the role of the meningeal lymphatics in the clearance of substances that come into parenchyma during opening of the BBB from the brain. The IHC, 2PLSM, and OCT data clearly show the increasing of the meningeal lymphatic vessels after opening of the BBB, which may indicate the activation of fluid movement in the brain.

\section{Conclusion}

Here, for the first time, we show the lymphatic drainage of the brain after opening of the BBB and successful application of OCT in imaging of the meningeal lymphatic vessels in the course of drainage functioning. These completely new fundamental results shed light on the mechanisms underlying the interaction between the meningeal lymphatics and the BBB function. This knowledge about the lymphatic drainage and brain clearance of metabolic waste products, as well as from substances that come to the brain tissues during the opening of the BBB, might give strong impulse for further studies in the diagnostics and therapy of CNS diseases associated with injuries of the BBB such as Alzheimer's disease, stroke, brain trauma, glioma, and depression.

\section{Disclosures}

Authors have no relevant financial interests in the manuscript and no other potential conflicts of interest to disclose.

\section{Acknowledgments}

The research performed in this work was supported by a grant from Russian Science Foundation No. 17-15-01263.

\section{References}

1. A. Louveau et al., "Structural and functional features of central nervous system lymphatic vessels," Nature 523(7560), 337-341 (2015).

2. A. Aspelund et al., "A dural lymphatic vascular system that drains brain interstitial fluid and macromolecules," J. Exp. Med. 212(7), 991-999 (2015).

3. P. Mascagni and G. B. Bellini, Istoria completa dei vasi linfatici, Vol. 2, Presso Eusebio Pacini e Figlio, Florence (1816). 
4. L. Belayev et al., "Quantitative evaluation of blood-brain barrier permeability following middle cerebral artery occlusion in rats," Brain Res. 739(1-2), 88-96 (1996).

5. D. Fernandez-Lopez et al., "Blood-brain barrier permeability is increased after acute adult stroke but not neonatal stroke in the rat," J. Neurosci. 32(28), 9588-9600 (2012).

6. O. Semyachkina-Glushkovskaya et al., "Stress plays provoking role in hypertension-related stroke: injuries of blood-brain barrier function," Proc. SPIE 10051, 100510I (2017).

7. E. I. Galanzha, V. V Tuchin, and V. P. Zharov, "Advances in small animal mesentery models for in vivo flow cytometry, dynamic microscopy, and drug screening," World J. Gastroenterol. 13(2), 192-218 (2007).

8. V. V. Tuchin, "In vivo optical flow cytometry and cell imaging," Riv. Nuovo Cimento Soc. Ital. Fis. 46(46), 375-416 (2014).

9. I. V. Fedosov et al., "Recording of lymph flow dynamics in microvessels using correlation properties of scattered coherent radiation," Quantum Electron. 32(11), 970-974 (2002).

10. I. V. Fedosov and V. V. Tuchin, "Bioflow measuring: laser Doppler and speckle techniques," in Handbook Coherent-Domain Optical Methods, V. V. Tuchin, Ed., pp. 487-563, Springer, New York (2013).

11. E. I. Galanzha et al., "In vivo fiber-based multicolor photoacoustic detection and photothermal purging of metastasis in sentinel lymph nodes targeted by nanoparticles," J. Biophotonics 2(8-9), 528-539 (2009).

12. V. V. Tuchin, A. Tárnok, and V. P. Zharov, "In vivo flow cytometry: a horizon of opportunities," Cytometry Part A 79A(10), 737-745 (2011).

13. S. Yousefi et al., "Label-free optical lymphangiography: development of an automatic segmentation method applied to optical coherence tomography to visualize lymphatic vessels using Hessian filters," J. Biomed. Opt. 18(8), 086004 (2013).

14. P. Gong et al., "In vivo label-free lymphangiography of cutaneous lymphatic vessels in human burn scars using optical coherence tomography," Biomed. Opt. Express 7(12), 4886-4898 (2016).

15. A. Joshi et al., "Molecular tomographic imaging of lymph nodes with NIR fluorescence," in 4th IEEE Int. Symp. on Biomedical Imaging: From Nano to Macro, pp. 564-567, IEEE (2007).

16. A. Zienkiewicz et al., "Continuous blood pressure recordings simultaneously with functional brain imaging: studies of the glymphatic system," Proc. SPIE 10063, 1006311 (2017).

17. O. Semyachkina-Glushkovskaya, "Silent vascular catastrophes in the brain in term newborns: strategies for optical imaging," IEEE J. Sel. Top. Quantum Electron. 22, 6802514 (2016).

18. A. Hoffmann et al., "High and low molecular weight fluorescein isothiocyanate (FITC)-dextrans to assess blood-brain barrier disruption: technical considerations," Transl. Stroke Res. 2(1), 106-111 (2011).

19. H.-L. Wang and T. W. Lai, "Optimization of Evans blue quantitation in limited rat tissue samples," Sci. Rep. 4, 6588 (2014).

20. D. E. Bragin et al., "High intracranial pressure effects on cerebral cortical microvasculr flow in rats," J. Neurotrauma 28, 775-785 (2011).

21. E. Caballero-Garrido et al., "In vivo inhibition of miR-155 promotes recovery after experimental mouse stroke," J. Neurosci. 35(36), 1244612464 (2015).

22. G. Egawa et al., "Intravital analysis of vascular permeability in mice using two-photon microscopy," Sci. Rep. 3, 1932 (2013).

23. S. Banerji et al., "Homodimerization of the lymph vessel endothelial receptor LYVE-1 through a redox-labile disulfide is critical for hyaluronan binding in lymphatic endothelium," J. Biol. Chem. 291(48), 25004-25018 (2016).

24. S. Banerji et al., "LYVE-1, a new homologue of the CD44 glycoprotein, is a lymph-specific receptor for hyaluronan," J. Cell Biol. 144(4), 789_ 801 (1999).

25. M. J. Karkkainen et al., "Vascular endothelial growth factor C is required for sprouting of the first lymphatic vessels from embryonic veins," Nat. Immunol. 5(1), 74-80 (2004).

26. O. Semyachkina-Glushkovskaya et al., "Laser speckle imaging and wavelet analysis of cerebral blood flow associated with the opening of the blood-brain barrier by sound," Chin. Opt. Lett. 15(9), 090002 (2017).

27. B. Mokri, "The Monro-Kellie hypothesis: applications in CSF volume depletion," Neurology 56(12), 1746-1748 (2001).

28. É. Mezey and M. Palkovits, "Neuroanatomy: forgotten findings of brain lymphatics," Nature 524(7566), 415-415 (2015).
29. M. W. Bradbury, H. F. Cserr, and R. J. Westrop, "Drainage of cerebral interstitial fluid into deep cervical lymph of the rabbit," Am. J. Physiol. 240(4), F329-F336 (1981).

30. H. F. Cserr, C. J. Harling-Berg, and P. M. Knopf, "Drainage of brain extracellular fluid into blood and deep cervical lymph and its immunological significance," Brain Pathol. 2(4), 269-276 (1992).

31. S. Kida, A. Pantazis, and R. O. Weller, "CSF drains directly from the subarachnoid space into nasal lymphatics in the rat: anatomy, histology and immunological significance," Neuropathol. Appl. Neurobiol. 19(6), 480-488 (1993).

Oxana Semyachkina-Glushkovskaya is a professor and chair of Physiology of Human and Animals at Saratov State University, Russia. Her research interests are focused on the study of nature of stress and development of stress-related vascular catastrophes in the brain and the application in practical medicine the innovative optical and mathematical technologies for prognosis and treatment of socially important stress-induced diseases.

Arkady Abdurashitov is a PhD student in the Department of Optics and Biophotonics at Saratov State University. His research interests include biomedical imaging, laser speckle contrast imaging, optical coherence tomography, data processing, and visualization. He successfully completed a Biophotonics and Imaging Graduate Summer School in Ireland.

Alexander Dubrovsky is a student in the Physics Faculty at Saratov State University. His research interests are in flow analysis by the means of optical modalities.

Denis Bragin is a Fellow of the American Heart Association and a member of the International Society for Cerebral Blood Flow and Metabolism, International Society for Oxygen Transport to Tissue, and American Heart/Stroke Association. His research interests include cerebral circulation and metabolism; physiological, biophysical and biochemical aspects of brain recovery with respect to cerebral ischemia, traumatic brain injury, and stroke.

Olga Bragina is a PhD student at the Department of Human and Animal Physiology at Saratov National Research State University. Her research interests include brain stimulation, brain pathology, histology, neurobehavioral, biomedical imaging, and laser speckle contrast imaging. She is a member of the Society for Neuroscience.

Natalia Shushunova is a student in the Department of Physiology of Humans and Animals in Saratov State University, Russia. Currently, she is in her fourth year and studies the function of the lymphatic system of stress influence.

Galina Maslyakova is a professor and head of Pathological Anatomy at Saratov State Medical University (SSMU). She is also the head of the Scientific Research Institute of Fundamental and Clinic Uronephrology at SSMU. She has authored of more than 50 peer-reviewed papers. She is a reviewer and an editor for the peer-reviewed journal Arkhiv Patologii. Her research interests include pathological anatomy, morphology, oncology, biomedical optics, and nanobiophotonics.

Nikita Navolokin graduated from SSMU in 2013. He is an assistant professor of pathological anatomy. He has authored 20 papers in peer-reviewed journals. From 2013 to present, he has been a researcher in the Laboratory of Cell Technologies of Scientific Research Institute of Fundamental and Clinic Uronephrology at SSMU. His research interests include pathological anatomy, morphology, oncology, drug delivery, biotechnology, biomedical optics, and nanobiophotonics.

Alla Bucharskaya received her $\mathrm{PhD}$ in pathology, oncology, and morphology of animals from Saratov State University (SSU), Saratov, Russia, in 1998. Her research interests include cytology, morphology and experimental oncology, biomedical optics, nanotheranostics, and nanobiophotonics. She has authored more than 30 peer-reviewed papers. She is a member of the Russian Association of Clinic Cytologists. Since 2010 to present she is head of the Centre of Collective Use in Scientific Research 
Institute of Fundamental and Clinic Uronephrology of Saratov State Medical University.

Valery Tuchin is a professor and head of optics and biophotonics at Saratov State University and several other universities and institutions. His research interests include tissue optics, laser medicine, tissue optical clearing, and nanobiophotonics. He is a fellow of SPIE and OSA, has been awarded Honored Science Worker of the Russia, SPIE Educator Award, FiDiPro (Finland), Chime Bell Prize of Hubei Province (China), and Joseph W. Goodman Book Writing Award (OSA/SPIE).

Jürgen Kurths is an academician of the European Academy of Sciences and a fellow of the American Physical Society. His research is related to complex systems theory, nonlinear dynamics as well as applications to the Earth, the human brain, the cardio-respiratory systems, which are characterized by a high degree of complexity and nonlinearity. $\mathrm{He}$ is a coauthor of more than 650 papers with more than 25,000 citations ( $h$-index $=90$ ).

Alexander Shirokov is a PhD senior researcher at the Laboratory of Immunochemistry, a head of the CKP Symbiosis, Institute of Biochemistry and Physiology of Plants and Microorganisms of the Russian Academy of Sciences. He is a chairman of the Council of Young Scientists of the Saratov Scientific Center of the Russian Academy of Sciences, and a member of the presidium of the Saratov Scientific Center of the Russian Academy of Sciences. 